Original Article

\title{
Patellar movement perception related to a backward-leaning standing position
}

\author{
Hitoshi Asai, RPT, PhD ${ }^{1)^{*}}$, Yuito Odashiro, RPT ${ }^{2)}$, Pleiades Tiharu InaOKa, RPT, PhD ${ }^{1)}$ \\ 1) Department of Physical Therapy, Graduate Course of Rehabilitation Science, School of Health \\ Sciences, College of Medical, Pharmaceutical, and Health Sciences, Kanazawa University: 5-11-80 \\ Kodatsuno, Kanazawa-shi, Ishikawa 920-0942, Japan \\ 2) Department of Physical Therapy, Medical Technology Part, Tokachi Rehabilitation Center, \\ Hokuto Social Medical Corporation, Japan
}

\begin{abstract}
Purpose] To investigate the patellar movement perception related to backward-leaning standing. [Subjects and Methods] Both the patellar range of motion during backward-leaning standing and perception related to patellar movement were analyzed using television-x irradiation images in 12 randomly selected healthy young individuals. [Results] Starting in a relaxed condition, two types of patellar movements were confirmed: those where the patella moves (patellar movement trials) and those where the patella does not move (patellar non-movement trials) during backward-leaning standing. The rate of the perceived patellar motion in the patellar movement trials was significantly higher $(90.9 \%)$ than that in the patellar non-movement trials $(66.7 \%)$. When starting in a quadricepscontracted condition, the rate of perceived trials (77.0\%) was significantly lower than that of the trials started in a relaxed condition. [Conclusion] The perception of patellar movement while backward-leaning standing may contribute to the perception of a backward standing position especially when the patella really moves.

Key words: Patella, Somatosensory, Backward standing position
\end{abstract}

(This article was submitted Mar. 30, 2017, and was accepted May 24, 2017)

\section{INTRODUCTION}

Positional perceptibility is considerably higher in positions where the center of pressure in the anteroposterior direction (CoPy position) is located farther from a quiet standing (QS) position, and lower in positions near the QS position ${ }^{1-3)}$. Standing position perceptibility in the anteroposterior direction (positional perceptibility) may be related to the standing posture stability ${ }^{1-3)}$. Such sensory information is believed to reference the internal model constructed in the higher neural centers for the positional perception of standing posture ${ }^{4}$.

Massion $^{5}$ postulated that a standing position is perceived through the relationship between the available sensory information and the body equilibrium, which has been memorized, based on experience. The perception of large changes in somatosensory information during forward and backward leaning has been investigated in previous studies ${ }^{6,7)}$. In the leaning standing position far from the QS position, the standing stability is low ${ }^{7}$, and both the leg muscle activity ${ }^{2}$ and foot pressure distribution $^{2,7)}$ are reported to differ considerably from those in the QS position. Therefore, large changes in the sensory information produced by changes in muscle activities and foot pressure distribution may provide important cues for standing position perception in the CoPy position.

The position of the center of pressure in the CoPy position during standing is represented as the percentage distance $(\% \mathrm{FL})$ from the hindmost point of the heel in relation to the foot length. A large increase in muscle activity is typically observed twice - at about 30-35\% FL and at about 25\% FL—while gradually leaning backward from the QS position ${ }^{6}$. Most

*Corresponding author. Hitoshi Asai (E-mail: asai@mhs.mp.kanazawa-u.ac.jp)

(C2017 The Society of Physical Therapy Science. Published by IPEC Inc.

(c) $(-)$ This is an open-access article distributed under the terms of the Creative Commons Attribution Non-Commercial No Deriva-

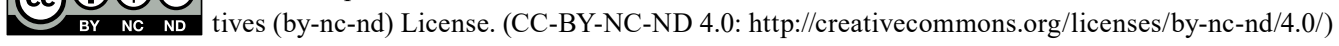


participants are unable to perceive the large increase in muscle activity of the rectus femoris and tibialis anterior muscles at $30-35 \% \mathrm{FL}^{6}$ ). Therefore, it is assumed that other sensory information in addition to the above-mentioned muscle activity are important for enhancing the standing position perception at the 30-35\% FL position. Given that the upward movement of the patella is observed at $30-35 \%$ FL when gradually leaning backward, we chose to focus on the perception of large changes in somatosensory information associated with the upward movement of the patella.

The patella is a sesamoid bone that has a connection to the quadriceps tendon, and the knee joint capsule surrounding the patella also adheres to the patellar tendon ${ }^{8,9}$. Consequently, it is assumed that the patellar tendon tension, the knee joint capsule, and the cutaneous tissues surrounding the knee change in association with upward patellar movement. Previous studies have investigated the contribution of somatosensory information from the soft tissue around the knee joint to the perception of knee joint movement ${ }^{10-14)}$. In particular, Edin ${ }^{14)}$ reported that sensory information from cutaneous receptors in the anterior thigh close to the knee joint play an important role in providing sensory or afferent information regarding the knee joint position. The rectus femoris muscle activity changes drastically in the standing position when gradually leaning backward from the QS position and is almost constant for every individual ${ }^{6)}$. Therefore, large changes in the somatosensory information produced by patellar movement and the quadriceps femoris muscle activation may contribute to the perception of a specific backward standing position.

In the present study, we sought to show that the patella moves in a vertical direction during backward leaning and to confirm the relationship between patellar movement and its perception. Our hypothesis was as follows: Participants whose patella moves clearly during backward standing also clearly perceive large changes in the somatosensory information associated with patellar movement.

\section{SUBJECTS AND METHODS}

A total of 12 subjects ( 3 females and 9 males) without neurological or orthopedic problems were randomly selected for this experiment. Their mean ( \pm standard deviation [SD]) age, height, weight, and foot length were $22.4 \pm 5.6$ years, 168.5 $\pm 6.4 \mathrm{~cm}, 65.5 \pm 7.8 \mathrm{~kg}$, and $25.4 \pm 1.2 \mathrm{~cm}$, respectively. All participants gave their informed consent to participate in this experiment, the protocol of which was approved by the institutional ethics committee of Kanazawa University in accordance with the Declaration of Helsinki (No. 229).

A television-x irradiation system (SHIMADZU Digital fluoroscopic system, SONICAL VISION Safire 2; Kyoto, Japan) was used to record the patellar active range of motion. The X-ray-generating power was $4.4 \mathrm{mGy} / \mathrm{min}$, and irradiation was delivered in the anterolateral position to the right knee. The elapsed time was superimposed on the obtained moving image with a video timer (FORA UTG-33, Tokyo, Japan), and the image was recorded to videotape using a video recorder (NVSX550, Panasonic, Kadoma, Japan). The frame rate of the recorder was set at $60 \mathrm{~Hz}$. The patellar movement onset timing was recorded using a miniature unidirectional accelerometer (AS-2G; KYOWA, Tokyo, Japan), which was taped to the ventral surface of the front-upper edge of the patella. The output from the accelerometer was amplified using an amplifier (DPM-611A; KYOWA Tokyo, Japan). A light switch (20 g) was held in the right hand, which generated an electrical signal in a rectangular waveform with $1.5 \mathrm{~V}$ of amplitude when activated.

All electrical signals were recorded on a computer (Dell Japan Inspiron 1300; Dell Japan, Kawasaki, Japan) via an A/D converter (ADA16-32/2[CB]F; Contec, Osaka, Japan) with a 1,000-Hz sampling rate and 16-bit resolution.

All participants were instructed to close their eyes and move their body with the ankle as the pivotal axis and to maintain the geometrical interrelationship among the body segments present at the QS position during standing backward leaning.

The participants maintained the QS posture for $3 \mathrm{~s}$ and then slowly moved from their initial standing position to the most backward-leaning standing position over about $10 \mathrm{~s}$. This experiment started after adequate backward-leaning movement training. The participants were instructed to activate the switch immediately when they felt some patellar movement while performing the backward-leaning movement. An experimenter wearing radiation protection stood behind the participant to prevent them from falling backward during the trial.

This experiment was conducted under two conditions: condition 1, in which the participants start with a relaxed quadriceps femoris (relax-start condition); and condition 2, in which participants start with a contracted quadriceps femoris, raising the patella superiorly as confirmed by observation and palpation (contract-start condition). Four trials were performed under each condition. X-ray exposure was performed under the relax-start condition to calculate the patellar range of motion (ROM). The participants were X-radiated during backward standing movement from QS to the most backward standing position in each trial. The total X-ray exposure time was within $1 \mathrm{~min}$, and the total exposure dose was under $4.4 \mathrm{mGy}$, ensuring a minimum amount of exposure.

Under the relax-start condition, the patella ROM was defined as the difference in the patellar position between the video image frame just before the beginning of the large fluctuation of the acceleration waveform and the frame right after the acceleration waveform convergence. The patellar position difference was calculated based on the distance (mm) from the upper edge of the tibia to the lower edge of the patella (Fig. 1). The mean and SD of the patellar ROM was calculated from four trials. The trials in which the patellar ROM exceeded the average value -2SD were defined as the patellar movement trials. The trials in which the patellar ROM was less than this value and the trials in which a patellar ROM was not observed were both defined as patellar non-movement trials. 


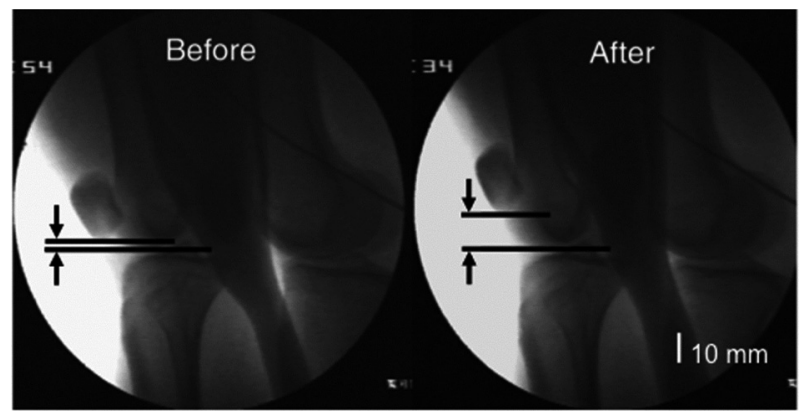

Fig. 1. Typical X-ray photographic image of the patellar movement Left side: before movement, Right side: after movement

Table 1. Results of perceptibility of patellar movement in the relax-start and the contract-start conditions

\begin{tabular}{|c|c|c|c|}
\hline & \multicolumn{2}{|c|}{ The relax-start condition } & \multirow[b]{2}{*}{$\begin{array}{l}\text { The contract-start } \\
\text { condition }\end{array}$} \\
\hline & $\begin{array}{c}\text { Patellar } \\
\text { movement trials }\end{array}$ & $\begin{array}{c}\text { Patellar } \\
\text { non-movement trials }\end{array}$ & \\
\hline Total trials & 33 & 15 & 48 \\
\hline Perceived traials & 30 & 10 & 37 \\
\hline Not perceived trials & 3 & 5 & 11 \\
\hline Rate of perceived trials (\%) & 90.9 & 66.7 & 77.0 \\
\hline
\end{tabular}

*Significant difference between patellar movement trials and patella non-movement trials under the relax-start condition.

The trials were also sub-divided into two categories: the trials where the switch was pressed (perceived trials) and the trials where it was not pressed (non-perceived trials). The rate of perceived trials for each category was then calculated.

In the relax-start condition, a $\chi^{2}$ goodness of fit test was used to evaluate the differences in the rate of perceived trials between the patella movement trials and the patella non-movement trials. The $\chi^{2}$ test was also used to assess the difference in the rate of perceived trials between the patella movement trials under the relax-start condition and the trials under the contract-start condition.

The alpha level was set at $\mathrm{p}<0.05$. All statistical analyses were performed using the SPSS $14.0 \mathrm{~J}$ software program (SPSS Japan, Tokyo, Japan).

\section{RESULTS}

Patellar movement during backward standing was confirmed in all 4 trials for 8 of the 12 participants. Three participants showed no patellar movement in all 4 trials, and 1 participant showed patellar movement in 3 trials and no patellar movement in 1 trial. There were 35 total trials with confirmed patellar movement, and the mean patellar ROM in these trials was $9.5 \mathrm{~mm}$ $(\mathrm{SD}=3.0 \mathrm{~mm})$. The trials in which the patellar ROM was less than $3.5 \mathrm{~mm}(9.5 \mathrm{~mm}-2 \mathrm{SD})$ were classified as non-movement trials. As a result of this classification, there were 33 patellar movement trials and 15 patellar non-movement trials (Table 1).

The perceived and non-perceived trials numbered 30 and 3, respectively, in the patellar movement trials. The rate of the perceived trials in the patellar movement trials was $90.9 \%$. However, in the patellar non-movement trials, the perceived and non-perceived trials numbered 10 and 5, respectively. The rate of perceived trials in the patellar non-movement trials was $66.7 \%$. There was a significant difference in the rate of perceived trials between the patellar movement and patellar nonmovement trials $\left(\chi^{2}(1, \mathrm{~N}=48)=17.53, \mathrm{p}<0.001\right)$ (Table 1).

The rate of perceived trials was $77.0 \%$ in the contract-start condition, which was significantly smaller than that of the perceived patellar movement trials under the relax-start condition $\left(\chi^{2}(1, \mathrm{~N}=48)=7.17, \mathrm{p}<0.01\right)$. However, no significant difference was recognized between this value and the rate of the perceived patellar non-movement trials (Table 1).

\section{DISCUSSION}

There were eight participants for whom patellar movement during backward leaning was confirmed in all four trials in the relax-start condition. There were three participants for whom patellar movement was not confirmed in any of the four 
trials. Therefore, two types of participants were confirmed in our study: those whose patella moved during backward leaning and those whose patella did not move during backward leaning. In the patellar non-movement trials, a slightly flexion of the knees or rectus femoris muscle contraction were visually confirmed in the participants from the quiet standing posture to the leaning posture. On the other hand, in the patellar movement trials, relaxed rectus femoris muscles were visually confirmed in the participants at the quiet standing posture. These differences in the rectus femoris activity and in the knee flexion angle at the starting position might cause the two patellar movement patterns.

The rate of the perceived trials under the relax-start condition was $91 \%$, which was significantly higher than that of the patellar non-movement trials under this condition. This indicates that a large change in the sensory information accompanying the patellar movement was perceived by a fairly high ratio of participants whose patella moved during backward leaning. In contrast, no significant difference was recognized between the rate of the perceived trials under the contract-start condition and that of the perceived trials in the patellar non-movement trials under the relax-start condition, suggesting that the contract-start condition probably simulated the patellar non-movement trials under the relax-start condition.

The misperception in patellar non-movement cases might have been caused by a large change in sensory information caused by changes in muscle activity intensity. This sensory information might include sensation from the rectus femoris and/or from the thigh skin movement due to stronger rectus femoris contraction. Therefore, even if there was no detectable movement of the patella in patellar non-movement cases, a patellar movement might be misperceived due to the sensory information produced by a large increase in muscle activity. A large increase in the rectus femoris activity is typically observed twice - at about 30-35\% FL and at about 25\% FL-while gradually leaning backward from the QS position ${ }^{6}$. A high percentage of participants were unable to perceive the $30-35 \%$ FL increase but were able to perceive the $25 \%$ FL increase $^{6)}$. The perception position in patellar non-movement cases may be significantly further backward than in the patellar movement trials.

The patella's mechanical role has been frequently investigated ${ }^{8,9)}$. However, little is known about patellar movement as a major source of sensory information regarding the standing position. The mean range of patellar movement is about $10 \mathrm{~mm}$. The joint capsule, the skin, and other soft tissues around the patella are deformed after receiving mechanical stimuli. Patellar movement is reportedly related to the rectus femoris activity, knee joint angle, and the load ${ }^{15-17)}$. Lateral patellar movement hardly occurs, at only $0^{\circ}$ to $10^{\circ}$ of knee flexion ${ }^{16}$ ). Therefore, we can ignore lateral patellar movement if the geometrical interrelationship among the body segments during QS is not largely changed. Proprioceptors within the joint capsules, ligaments, skin, and muscles provide information about the joint position and movement contributing to positional perception ${ }^{10-14,18-21)}$.

There are many kinds of soft tissues around the patella: the joint capsule, the patellar retinaculum, the patellar tendon, skin, and the quadriceps femoris ${ }^{9}$. Thus, based on the findings of previous studies, patellar movement produces sensory information changes by increasing the discharge frequency of mechanoreceptors within the joint capsule, ligament, skin and muscles via mechanical deformation and rectus femoris activation. In cases when the patella moves while leaning backward, the above-cited references suggest that the patellar movement is perceived accurately through large changes in the sensory information obtained via mechanoreceptors within the soft tissues around the patella, which supports our results.

The present findings suggest that the large changes in the sensory information by patellar movement are a cue for the perception of the standing position while leaning backward.

Only four trials were performed under the relax-start condition in order to minimize the radiation exposure to the participants in this study. Furthermore, these four trials were executed under the contract-start condition in order to match the trial number under the relax-start condition. This study focused on the movement of the patella while in the backward-leaning standing position. Therefore, X-ray images and an accelerometer taped to the ventral surface of the front-upper edge of the patella were used to detect the patellar movement. An investigation of the relationship between the movement of the patella and muscle activity using electromyography from the rectus femoris is needed in future.

This investigation identified the patellar movement perception related to backward-leaning standing using television- $\mathrm{x}$ irradiation images and an accelerometer. Starting from the relaxed condition, two patterns involving the patella were confirmed: one in which the patella moves, and another in which the patella does not move during backward-leaning standing. The rate of the perceived patellar motion in the patellar movement trials was significantly higher than that in the patellar nonmovement trials. When starting from the quadriceps-contracted condition, the rate of perceived trials was significantly lower than in the trials started in a relaxed condition. The perception of patellar movement while performing a backward-leaning motion while standing may contribute to the perception of the backward-leaning position.

\section{ACKNOWLEDGEMENTS}

This work was supported by JSPS KAKENHI, Grant-in-Aid for Scientific Research (C), Grant Number 23300238. The author would like to thank Professor Kichiro Koshida, Associate Professor Kosuke Matsubara (Department of Quantum Medical Technology, Faculty of Health Sciences, Kanazawa University), and the members of the radiology division of Kanazawa University Hospital for their radiographic work. 


\section{REFERENCES}

1) Fujiwara K, Asai H, Toyama H, et al.: Perceptibility of body position in anteroposterior direction while standing with eyes closed. Percept Mot Skills, 1999, 88: 581-589. [Medline] [CrossRef]

2) Fujiwara K, Asai H, Miyaguchi A, et al.: Perceived standing position after reduction of foot-pressure sensation by cooling the sole. Percept Mot Skills, 2003, 96: 381-399. [Medline] [CrossRef]

3) Fujiwara K, Asai H, Kiyota N, et al.: Relationship between quiet standing position and perceptibility of standing position in the anteroposterior direction. J Physiol Anthropol, 2010, 29: 197-203. [Medline] [CrossRef]

4) Gurfinkel VS, Levic FS: Brain and space. New York: Oxford University Press, 1991.

5) Massion J: Movement, posture and equilibrium: interaction and coordination. Prog Neurobiol, 1992, 38: 35-56. [Medline] [CrossRef]

6) Asai H, Fujiwara K: Perceptibility of large and sequential changes in somatosensory information during leaning forward and backward when standing. Percept Mot Skills, 2003, 96: 549-577. [Medline] [CrossRef]

7) Fujiwara K, Asai H, Koshida K, et al.: Perception of large change in distribution of heel pressure during backward leaning. Percept Mot Skills, 2005, 100: 432-442. [Medline] [CrossRef]

8) Kapandji IA: Physiology of the joints. Lower Limb Volume 2, 5th ed. New York: Elsevier Health Sciences, 1987.

9) Standring S: Gray's Anatomy. The anatomical basis of clinical practice, expert consult, 40th ed. Philadelphia: Churchill Livingstone, 2008.

10) Barrack RL, Skinner HB, Brunet ME, et al.: Functional performance of the knee after intraarticular anesthesia. Am J Sports Med, 1983, 11: 258-261. [Medline] [CrossRef]

11) Barrack RL, Skinner HB, Cook SD, et al.: Effect of articular disease and total knee arthroplasty on knee joint-position sense. J Neurophysiol, 1983, 50: 684-687. [Medline]

12) Clark FJ, Horch KW, Bach SM, et al.: Contributions of cutaneous and joint receptors to static knee-position sense in man. J Neurophysiol, 1979, 42 : $877-888$. [Medline]

13) Collins DF, Refshauge KM, Todd G, et al.: Cutaneous receptors contribute to kinesthesia at the index finger, elbow, and knee. J Neurophysiol, 2005, 94: 1699-1706. [Medline] [CrossRef]

14) Edin B: Cutaneous afferents provide information about knee joint movements in humans. J Physiol, 2001, 531: 289-297. [Medline] [CrossRef]

15) Brunet ME, Brinker MR, Cook SD, et al.: Patellar tracking during simulated quadriceps contraction. Clin Orthop Relat Res, 2003, 414: 266-275. [Medline] [CrossRef]

16) Draper CE, Besier TF, Fredericson M, et al.: Differences in patellofemoral kinematics between weight-bearing and non-weight-bearing conditions in patients with patellofemoral pain. J Orthop Res, 2011, 29: 312-317. [Medline] [CrossRef]

17) Li G, Papannagari R, Nha KW, et al.: The coupled motion of the femur and patella during in vivo weightbearing knee flexion. J Biomech Eng, 2007, 129: 937-943. [Medline] [CrossRef]

18) Isaac SM, Barker KL, Danial IN, et al.: Does arthroplasty type influence knee joint proprioception? A longitudinal prospective study comparing total and unicompartmental arthroplasty. Knee, 2007, 14: 212-217. [Medline] [CrossRef]

19) Kavounoudias A, Roll R, Roll JP: Foot sole and ankle muscle inputs contribute jointly to human erect posture regulation. J Physiol, 2001, 532: 869-878. [Medline] [CrossRef]

20) McCloskey DI, Cross MJ, Honner R, et al.: Sensory effects of pulling or vibrating exposed tendons in man. Brain, 1983, 106: 21-37. [Medline] [CrossRef]

21) Roll R, Kavounoudias A, Roll JP: Cutaneous afferents from human plantar sole contribute to body posture awareness. Neuroreport, 2002,13 : $1957-1961$. [Medline] [CrossRef] 